\title{
Electrosprayed Nanoparticle Delivery System for Controlled Release
}

\author{
Megdi Eltayeb $^{\mathrm{a}}$, Eleanor Stride ${ }^{\mathrm{b}}$, Mohan Edirisinghe ${ }^{\mathrm{c}}$, Anthony Harker ${ }^{\mathrm{e}, *}$ \\ ${ }^{a}$ Department of Biomedical Engineering, Sudan University of Science and Technology, Khartoum, Sudan, PO Box 407 \\ ${ }^{b}$ Institute of Biomedical Engineering, Department of Engineering Science, University of Oxford, Old Road Campus Research \\ Building, Headington $O X 37 D Q, U K$ \\ ${ }^{c}$ Department of Mechanical Engineering, University College London, Torrington Place, London, WC1E 7JE, UK \\ ${ }^{d}$ London Centre for Nanotechnology, Gordon Street, London, WC1H OAH, UK \\ ${ }^{e}$ London Centre for Nanotechnology and Department of Physics 89 Astronomy, University College London, Gower Street, \\ London, WC1E 6BT, UK
}

\begin{abstract}
This study utilises an electrohydrodynamic technique to prepare core-shell lipid nanoparticles with a tunable size and high active ingredient loading capacity, encapsulation efficiency and controlled release. Using stearic acid and ethylvanillin as model shell and active ingredients respectively, we identify the processing conditions and ratios of lipid:ethylvanillin required to form nanoparticles. Nanoparticles with a mean size ranging from 60 to $70 \mathrm{~nm}$ at the rate of $1.37 \times 10^{9}$ nanoparticles per minute were prepared with different lipid:ethylvanillin ratios. The polydispersity index was $\approx 21 \%$ and the encapsulation efficiency $\approx 70 \%$. It was found that the rate of ethylvanillin release was a function of the nanoparticle size, and lipid:ethylvanillin ratio. The internal structure of the lipid nanoparticles was studied by transmission electron microscopy which confirmed that the ethylvanillin was encapsulated within a stearic acid shell. Fourier transform infrared spectroscopy analysis indicated that the ethylvanillin had not been affected. Extensive analysis of the release of ethylvanillin was performed using several existing models and a new diffusive release model incorporating a tanh function. The results were consistent with a core-shell structure.
\end{abstract}

Keywords: Electrohydrodynamic processing, controlled release, lipid nanoparticles

\section{Introduction}

Nanotechnology has been widely utilised for encapsulation and controlled release of active ingredients in the pharmaceutical, cosmetic, and food industries in recent years[1-8]. For these applications the nanoparticles must be stable, nontoxic, have high encapsulation efficiency and loading capacity and enable the release kinetics of the flavour component to be tailored to meet specific requirements $[9,10]$. Lipid-based colloidal particles meet these criteria and there are several different classes, e.g. solid lipid nanoparticles, liposomes and micelles[11, 12]. They have a wide spectrum of applications, for example encapsulation of flavour, preservatives, vitamins and

\footnotetext{
*Corresponding Author

Email addresses: megdi.eltayeb@sustech.edu (Megdi Eltayeb), eleanor.stride@eng.ox.ac.uk (Eleanor Stride), m.edirisinghe@ucl.ac.uk (Mohan Edirisinghe), a.harker@ucl.ac.uk (Anthony Harker)
}

other so called "nutraceuticals" [1, 11, 13, 14]. They are effective carriers for both lipophilic and hydrophilic flavour components, and have been shown to increase bioavailability as compared with oral administration of unencapsulated material[1518]. Several liposome-containing food ingredients have already been approved for use or entered trial development[19-22]. The limitations of liposomes for food encapsulation, however, include a relatively low loading capacity, rapid flavour component release, and instability in storage[23]. Solid lipid nanoparticles, consisting of a matrix of solid lipid as opposed to a lipid lamellar structure surrounding a liquid core, have been shown to exhibit greater stability and more sustained release profiles [24, 25].

There are three main strategies for flavour encapsulation reported in the literature: (i) Homogeneous matrix, (ii) Flavour component enriched shell, and (iii) Flavour component enriched core. 
In homogeneous matrix particles the flavour component is dispersed uniformly throughout the particle. This allows for prolonged flavour release (over weeks) and hence protection against oxidation $[26$, 27], and high encapsulation efficiency[24, 28]. Particles with flavour component enriched shells conversely are characterised by very rapid release rates (over minutes)[29, 30]. Particles with flavour component enriched cores exhibit release rates governed by Ficks law of diffusion[31] and dependent upon the physicochemical properties (i.e. partition coefficient) of the flavour component molecules and the thickness and density of the outer lipid shell[32, 33].

A range of different techniques has been utilised to prepare solid lipid nanoparticles. There are two main classes[24, 26]: hot homogenisation and cold homogenisation. The former provides poor control over the physical and chemical properties of the solid lipid nanoparticles and loss of the active ingredient to the surrounding medium and/or through recrystallisation. Cold homogenisation however favours the formation of particles with flavour component enriched shells[8]. It is desirable therefore to explore alternative methods for fabricating solid lipid nanoparticles that have the potential to overcome these drawbacks. This study examines the use of electrohydrodynamic techniques for this application. Electrohydrodynamic atomisation or electrospraying is the process of generating fine droplets ranging from micrometres to nanometres in diameter from the electrically induced focusing and breakup of a liquid jet[34-36]. The size and uniformity of the particles generated depends principally upon the physical properties of the materials being processed, their flow rate(s) and the applied electrical potential difference[37, 38].

Electrohydrodynamic techniques have been used to produce nano- and micro-scale structures for a wide range of applications[39-49]. They offer excellent control over particle size, structure and uniformity and do not require the use of multiple processing steps or elevated temperature or pressure. It has also been shown recently that multilayer particles can be generated using both single[50, 51] and co-axial spraying devices[52-54].

In this work, electrohydrodynamic processing was used to form stearic acid (SA) nanoparticles encapsulating ethylvanillin (EV). Both materials are widely used in the food industry[55] and are representative of typical hydrophobic coatings and hydrophilic flavour components respectively. The aim in this paper was to undertake a detailed char- acterization of the rate of release of active component as this is of great importance in tuning particle characteristics to a particular application. Thus, as well as demonstrating that our techniques[50, 51] are applicable to a wider range of materials combinations, we have significantly improved our analysis of the release kinetics.

\section{Materials and methods}

\subsection{Materials}

Stearic acid, ethylvanillin (3-ethoxy-4hydroxybenzaldehyde), and 95\% (v) ethanol were all purchased from Sigma-Aldrich (Poole, Dorset, UK). Doubled distilled (DD) water was used as the release medium in all the experiments.

\subsection{Preparation and characterisation of spraying solutions}

Stearic acid and ethylvanillin were dissolved in ethanol with concentrations ranging from 1 to $4 \mathrm{wt} \%$ and (Table 1). The solutions were mixed using a magnetic stirrer at ambient temperature $\left(25^{\circ} \mathrm{C}\right)$ until optically transparent: this took about 10 minutes. Surface tension and viscosity were obtained for each solution from three repeat measurements by the plate method using a Krüss tensiometer (Model-K9, Krüss GmbH, Germany) and U-tube viscometer (75 ml Cannon-Fenske Routine Viscometer, Cannon Instruments, USA), respectively.

\subsection{Electrohydrodynamic processing}

The solutions were fed through silicone tubing from a $10 \mathrm{ml}$ plastic syringe driven by a high precision syringe pump (Harvard PHD 4400, Edenbridge, UK) into a stainless steel needle with internal diameter $450 \mu \mathrm{m}$, at a flow rate of $15 \mu \mathrm{lmin}^{-1}$. The applied electrical potential difference between the needle and a ground electrode was varied between 13 and $15 \mathrm{kV}$, using a high voltage power supply (Glassman Europe Ltd., Tadley, UK). Once a stable cone jet had been obtained, at an optimal voltage of $14.5 \mathrm{kV}$, nanoparticles were collected either on a glass microscope slide, or in a vial containing DD water for the release studies, both of which were kept at distance of $\sim 100 \mathrm{~mm}$ from the needle tip. The jet created at the tip of the needle during nanoparticle production was observed using a video camera (Leica S6D JVC-color). Optimisation of the operating parameters and formulation 
with regard to their influence on nanoparticle physical properties and ethylvanillin release rate was carried out prior to this work $[50,56]$ and the operating parameters for this study (i.e. lipid concentration, solution flow rate, distance between needle tip and collector) were selected in order to produce a stable cone jet and controlled nanoparticle formation $[50,56]$.

\subsection{Scanning electron microscopy (SEM)}

To characterise the size and morphology of the lipid nanoparticles, they were examined using SEM (Model JEOL JSM 3600, UK). Dried lipid nanoparticles were vacuum sputter coated with gold for $2 \mathrm{~min}$, at $40 \mathrm{~mA}$, and mounted on aluminium stubs with double sided carbon tape, prior to SEM examination, which was carried out at $5 \mathrm{kV}$. ImageJ software (National Institutes of Health NIH, Maryland, USA) was used to measure the standard deviation and mean of the nanoparticle diameter. Approximately 300 nanoparticles were measured for each set of processing conditions.

Using the particle diameters to compute the average particle volume $V_{\mathrm{np}} \mathrm{m}^{3}$, the number of particles produced per minute, $R$, was calculated from

$$
R=\frac{V_{\mathrm{EVA}}+V_{\mathrm{SA}}}{V_{\mathrm{EVA}}+V_{\mathrm{SA}}+V_{\mathrm{EtOH}}} \frac{F}{V_{\mathrm{np}}},
$$

where $V_{\mathrm{EVA}}, V_{\mathrm{SA}}$ and $V_{\mathrm{EtOH}}$ are the volumes of ethylvanillin, stearic acid, and ethanol in a unit volume of the spraying fluid and $F$ is the flow rate of the fluid in $\mathrm{m}^{3}$ minute ${ }^{-1}$.

\subsection{Transmission electron microscopy (TEM)}

To characterise the internal structure of the lipid nanoparticles, they were observed by TEM (JEOL1010, Tokyo, Japan) with negative staining of the lipid to improve their electron density with 2wt\% solution of uranyl acetate for $30 \mathrm{~s}$ and allowed to dry. Analysis of the stained grids was carried out at an accelerating voltage of $80 \mathrm{kV}$.

\subsection{X-ray diffraction}

The powder X-ray diffraction (XRD) pattern of the nanoparticle samples was determined using a Thermo-ARL Xtra, Thermo Scientific diffractometer using $\mathrm{CuK} \alpha 1$ radiation $(\lambda=1.542 \AA)$. The generator voltage was set at $40 \mathrm{kV}$ and the current at $30 \mathrm{~mA}$. The nanoparticle sample was scanned over the $2 \theta$ angular range $3^{\circ}$ to $40^{\circ}$ with a step size of $0.02^{\circ}$ and a scanning speed of $4^{\circ} \mathrm{min}^{-1}$.

\subsection{Fourier transform infrared (FTIR) spec- troscopy}

Characterisation of lipid nanoparticle structure was performed using Fourier Transform Infrared (FTIR) Spectroscopy (Perkin-Elmer, 2000 FTIR spectrometer, Cambridge, UK). $2 \mathrm{mg}$ samples of powdered lipid nanoparticles were mixed with Potassium Bromide ( $\mathrm{KBr}$ ) and hydraulically pressed to form pellets. Measurements were taken over the range of $400-4000 \mathrm{~cm}^{-1}$, averaging 256 scans at a resolution of $1 \mathrm{~cm}^{-1}$ for each run, at the ambient temperature $\left(25^{\circ} \mathrm{C}\right)$.

\subsection{Determination of ethylvanillin encapsulation efficiency, loading capacity and release profile}

To measure the ethylvanillin encapsulation efficiency, loading capacity and release profile of ethylvanillin from nanoparticles made from each solution, $3 \mathrm{ml}$ of the collected suspensions were split equally into 3 tubes. These tubes were filtered through $200 \mathrm{~nm}$ syringe filters in $10 \mathrm{ml}$ of DD water at the ambient temperature $\left(25^{\circ} \mathrm{C}\right)$. Lipid nanoparticles from three tubes were obtained individually and mixed with an equal amount of DD water to disperse the lipid nanoparticles. The resulting free ethylvanillin content in each tube was examined using ultraviolet (UV) spectrophotometry (Perkin Elmer, Lambda 35, UV/Vis spectrophotometer, Waltham, USA). Ethylvanillin absorbance was measured by a UV spectrophotometry detector at $278 \mathrm{~nm}$. The amount of ethylvanillin encapsulated in the lipid nanoparticles was calculated by subtracting the amount of ethylvanillin in the DD water from the total amount of the ethylvanillin in the lipid nanoparticle solution. Encapsulation efficiency (EE) and loading capacity (LC) were determined using equations 1 and 2 respectively.

$$
\begin{aligned}
& \mathrm{EE}=\frac{\mathrm{EV}_{\mathrm{np}}-\mathrm{EV}_{\mathrm{sn}}}{\mathrm{EV}_{\mathrm{sn}}} \times 100 \% \\
& \mathrm{LC}=\frac{\mathrm{NP}}{\mathrm{DNP}} \times 100 \%
\end{aligned}
$$

where $\mathrm{EV}_{\mathrm{np}}$ is the weight of ethylvanillin in the nanoparticles, $\mathrm{EV}_{\mathrm{sn}}$ is the weight of ethylvanillin in the supernatant, NP is the total weight of lipid nanoparticles and DNP is the total weight of dried electrosprayed nanoparticles. Release studies were conducted over 240 mins and repeated three times for each set of lipid nanoparticles and the results combined to obtain the cumulative ethylvanillin release rate, which was plotted as a function of time. 
In addition, the ethylvanillin release rate profile data were fitted using Higuchi, zero-order, HixsonCrowell, first-order, and Ritger-Peppas models, respectively[57-59] and equations for the released fraction are given below. The zero-order expression is

$$
Q_{t}=K_{0} t
$$

where $Q_{t}$ is the proportion of ethylvanillin released in time $t$, and $K_{0}$ is the zero order release constant with units of inverse time.

The first order expression is

$$
Q_{t}=Q_{\infty}\left(1-e^{-K_{1} t}\right)
$$

where $Q_{\infty}$ is the total fraction of ethylvanillin released from the nanoparticles, $Q_{t}$ is the proportion released in time $t$ and $K_{1}$ is the release constant. In a simple quasistatic model of release in which a sphere of radius $a$ in which the concentration does not vary with position is surrounded by a membrane of thickness $\delta$ and diffusion constant $D$ we may identify $K_{1}$ with $3 D / a \delta$.

Higuchi's expression is

$$
Q_{t}=K_{\mathrm{H}} t^{1 / 2}
$$

where $Q_{t}$ is the proportion of ethylvanillin released in time $t$, and $K_{\mathrm{H}}$ is the Higuchi dissolution constant.

The Hixson-Crowell model gives

$$
Q_{t}=Q_{\infty}\left(1-(1-\alpha t)^{3}\right)
$$

where $Q_{\infty}$ is the total fraction of the ethylvanillin released from the nanoparticles, $Q_{t}$ is the fraction of ethylvanillin released in time $t$ and $\alpha=9 K_{\mathrm{HC}} / r_{0}$ depends on the release constant for Hixson-Crowell release, $K_{\mathrm{HC}}$ and the initial radius $r_{0}$.

Peppas and collaborators give a power-law release rate, applicable only to the first $60 \%$ of the release,

$$
Q_{t}=A t^{n}
$$

where $Q_{t}$ is the fraction of ethylvanillin released at time $t, A$ is a constant incorporating geometric and structural features of the nanoparticles, and $n$ is the release exponent that indicates the release rate mechanism. The value of $n$ is 0.45 for a Fickian diffusion mechanism, 0.89 for so-called Case II release mechanism, and $n>0.45$ and $n<0.89$ for a nonFickian diffusion mechanism; $n>0.89$ for Super Case II release mechanism[60].
In order to distinguish between different models of the particle structure we needed a model for diffusive release from a homogeneous particle which could be applied across the whole release process not one which not limited to a part of the process or which had to be artificially truncated to limit the release to $100 \%$. We therefore developed an expression (see Appendix) based on the same diffusive release model as used by Peppas and collaborators but applicable to the whole of the release process:

$$
Q_{t}=Q_{\infty} \tanh \left(\alpha t^{1 / 2}\right)
$$

where $Q_{\infty}$ is the total fraction of ethylvanillin released from the nanoparticles, $Q_{t}$ is the fraction released in time $t$ and $\alpha$ is a constant which may be related to the particle size and diffusion constant.

We used the NonlinearModelFit function of Mathematica(c), and assessed the goodness of fit by considering the $R^{2}$ values, the adjusted $R^{2}$ which takes account of the number of fitting parameters, and the size-corrected Akaike Information Criterion, $\mathrm{AICc}$

\section{Results and discussion}

In this work, we used a lipid (stearic acid) to encapsulate ethylvanillin using electrohydrodynamic technology. Since stearic acid is a natural lipid extracted from animal fat; we expect the lipid nanoparticles should be safe and biocompatible for food applications. As showed in Figure 1a, nanoparticles were successfully prepared using a single step electrohydrodynamic technique at a flow rate of $15 \mu \mathrm{lmin}^{-1}$ with an applied voltage of $14.5 \mathrm{kV}$ and a collection distance of $\sim 100 \mathrm{~mm}$. Uniform nanoparticles with a mean diameter of $65 \pm 6 \mathrm{~nm}$ (Figure $1 \mathrm{~b}, \mathrm{c}$ ) at the rate of $1.37 \times 10^{9}$ nanoparticles per minute were obtained. Lipid assembles around the ethylvanillin core to form a shell (Figure 1d). TEM examination indicated an $\mathrm{EV}$ rich core surrounded by an SA rich outer layer (Figure 1e). The concentration of SA will change the solution viscosity, surface tension and electrical conductivity; this will in turn alter the behaviour of the jet and hence the characteristics of the nanoparticles produced[37].

As shown in Figure 2 (a, b), varying the lipid weight ratio from 1 to $4 \mathrm{wt} \%$ to encapsulate $1 \mathrm{wt} \%$ ethylvanillin solution, viscosity (0.82 to $1.18 \mathrm{mPas}$ ) and surface tension (21.4 to $22.1 \mathrm{mN} \mathrm{m}^{-1}$ ) results in nanoparticles with diameters from $52-65 \mathrm{~nm}$, 


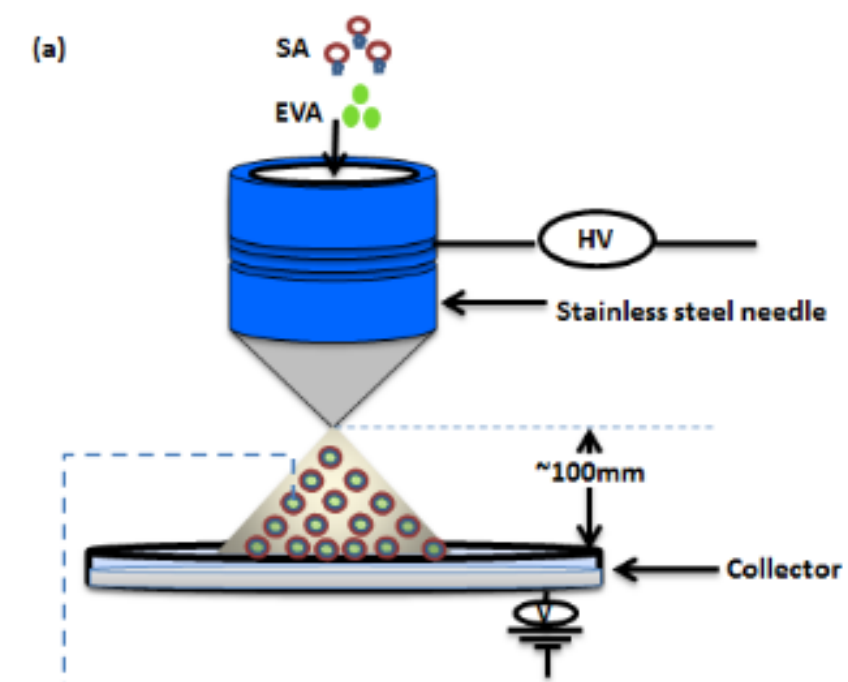

(b)

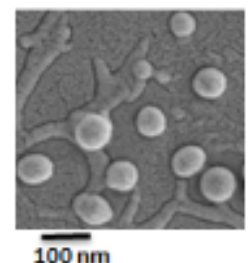

(c)

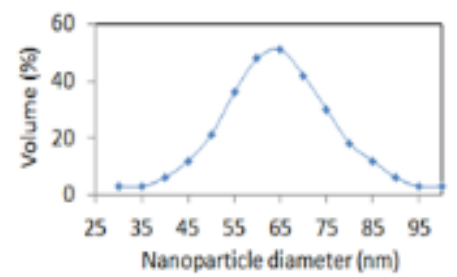

(d)

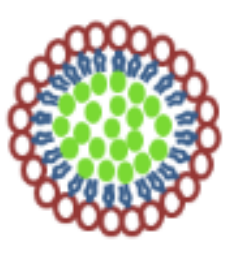

(e)

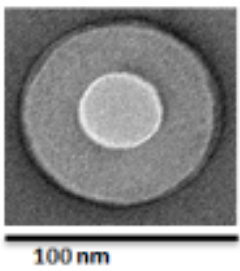

Figure 1: Schematic illustration; (a) EHD set-up. (b) SEM image of $4 \mathrm{wt} \%$ stearic acid lipid nanoparticles encapsulating $1.6 \mathrm{wt} \% \mathrm{EV}$ in their structure generated at an applied voltage of $14.5 \mathrm{kV}$ and flow rate of $15 \mu \mathrm{lmin} \mathrm{m}^{-1}$. (c) Size distributions of nanoparticles containing EV. ( $\mathrm{d}$ and e) schematic and corresponding TEM image of a nanoparticle. Average size is $65 \pm 6 \mathrm{~nm}$. Scale bar $100 \mathrm{~nm}$. 
for ethylvanillin delivery application $[50,56]$. Keeping the SA weight constant at $4 \mathrm{wt} \%$, we varied the ethylvanillin weight concentration ratio from 1 to $4 \mathrm{wt} \%$, decreasing the viscosity and surface tension and revealed that we can tune the nanoparticle size in a reproducible manner[61]. For example, when the viscosity of the SA-EV solution was decreased from 1.18 to $0.98 \mathrm{mPas}$, the nanoparticle size decreased from $65 \pm 7 \mathrm{~nm}$ (polydispersity $=21 \%, N=300$ ) to $58 \pm 10 \mathrm{~nm}$ (polydispersity = $33 \%, N=300$ ) (Figure 2b), while the nanoparticle solution surface tension only slightly fluctuated in the range of 22.1 to $21.8 \mathrm{mN} \mathrm{m}^{-1}$ (Figure $2 \mathrm{c}, \mathrm{d}$ ) [6164]. The observed influence of ethylvanillin inherent viscosity on the solution was consistent with what has previously been reported for stearic acid-ethyl cellulose hybrid nanoparticles[50].

The X-ray diffraction (XRD) patterns obtained are shown in Figure 3. The XRD pattern for the ethylvanillin loaded lipid nanoparticles (solid line) is very similar to the peaks assigned to a standard lipid crystal structure (short-dashed line). As the XRD pattern of the ethylvanillin loaded lipid nanoparticles did not show any peak corresponding to the ethylvanillin crystal structure (long-dashed line) we conclude that pure ethylvanillin is present in the centre and pure SA at the surface of the particle, which further confirms our interpretation of the particle structure.

The FTIR spectrum of EV (long-dashed line in Figure 4) shows peaks at 3366, 2918, 2850, and $1701 \mathrm{~cm}^{-1}$ which belong to phenolic $(-\mathrm{O}-$ $\mathrm{H})$ stretching, asymmetric stretching of alkenes $(-\mathrm{CH}-)$ of the $\mathrm{OCH}_{2}$ group, aromatic $\mathrm{C}=\mathrm{C}$ stretching vibration [65] and the $\mathrm{C}=\mathrm{O}$ carbonyl stretching vibration[66], respectively. In addition to this, peaks at $\sim 1111 \mathrm{~cm}^{-1}$ were seen, belonging to $\mathrm{C}-\mathrm{O}$ stretching and at $524 \mathrm{~cm}^{-1}$ corresponding to vibration and bending[67]. The spectrum of SA (short-dashed line in Figure 4) shows two strong absorption peaks at $2953 \mathrm{~cm}^{-1}$ and $2852 \mathrm{~cm}^{-1}$ that are attributed to the asymmetric and symmetric stretching vibrations of its $-\mathrm{CH}_{2}$ group. The strong absorption peak at $1711 \mathrm{~cm}^{-1}$ corresponds to the stretching vibration of the carbonyl $\mathrm{C}=\mathrm{O}$ group. The absorption peaks around $1471 \mathrm{~cm}^{-1}$ represent the bending vibration of $-\mathrm{CH}_{3}$ groups. The absorption peaks from $1310 \mathrm{~cm}^{-1}$ to $1105 \mathrm{~cm}^{-1}$ represent the rocking vibration of the $-\mathrm{CH}_{2}$ group. The nanoparticle spectra (solid line in Figure 4) exhibited peaks that were a combination of the characteristic peaks obtained with pure EV and pure SA. However, the $\mathrm{C}=\mathrm{O}$ band shifted from 1701 to $1711 \mathrm{~cm}^{-1}$, which indicates hydrogen bonding formed between $\mathrm{O}-\mathrm{H}$ and $\mathrm{C}-\mathrm{H}$ in $\mathrm{EV}$ within $\mathrm{SA}$. This indicates that there was no degradation or chemical interaction which occurred between EV and SA.

Next, we examined the EV loading capacity, encapsulation efficiency, and release profile from the SA lipid nanoparticles prepared from solutions with different SA concentrations from 1 to $4 \mathrm{wt} \%$. As shown in Figure 5a, for nanoparticles prepared from the EV: SA $1: 4$ solution, $71 \pm 3 \%$ of $\mathrm{EV}$ was encapsulated into the nanoparticles. In contrast, EV encapsulation efficiencies for the nanoparticles prepared from 1, 2 and $3 \%$ solutions were $48 \pm 3$, $55 \pm 4$ and $62 \pm 6 \%$, respectively. Increasing the ethylvanillin content of the spraying solution eventually led to unstable jets, which may explain the non-monotonic variation of loading capacity with ethylvanillin content shown in Figure 5c. Figure $5 \mathrm{~b}$ shows the how the release of the $\mathrm{EV}$ varied over $240 \mathrm{~min}$. The $4 \mathrm{wt} \%$ lipid nanoparticles release $\sim 5 \%$ of EV over $60 \mathrm{~min}$ compared to 12,20 and 48 mins for 1, 2, $3 \mathrm{wt} \%$ lipid nanoparticles, respectively. These results further indicate that the SA at the interface of the core acts as a shell that helps to protect the ethylvanillin inside the nanoparticles.

We further observed that the EV loading capacities of these nanoparticles were relatively high. For instance, when the EV concentration was 1, 2 and $3 \mathrm{wt} \%$, the measured loading capacity reached 0.6, 1.2 , and $1.6 \%$ respectively (Figure $5 \mathrm{c}$ ). However, EV loading capacity decreased when the initial EV input was increased to $\sim 4 \mathrm{wt} \%$. We also observed that EV was released from the nanoparticles in a controlled manner until its loading capacity was higher than $\sim 1.6 \%$ (Figure $5 \mathrm{~d}$ ).

Lastly, we examined which release model gave the best fit to the experimental results. The models which performed best were the tanh function and the first-order model, and the results are summarised in Table 1 (note that a better fit is denoted by a larger $R^{2}$ or $R_{\text {adj }}^{2}$, but by a smaller AICc).

Examining the parameters which result from the fit, however, we find that the parameter $\alpha$ from the $\tanh (\alpha \sqrt{t})$ fit does not scale as $1 / d$ as we would expect for diffusion of an active ingredient from a solid particle. This suggests that we should reject this model. The first-order model, however, yielded parameters $K_{1}$ which could be fitted very well by $K_{1}=3 D /((\langle d\rangle / 2-\delta) \delta)$ with $D=1.2 \times 10^{-18} \mathrm{~m}^{2} \mathrm{~s}^{-1}$ and $\delta=22 \times 10^{-9} \mathrm{~m}$. 
(a)
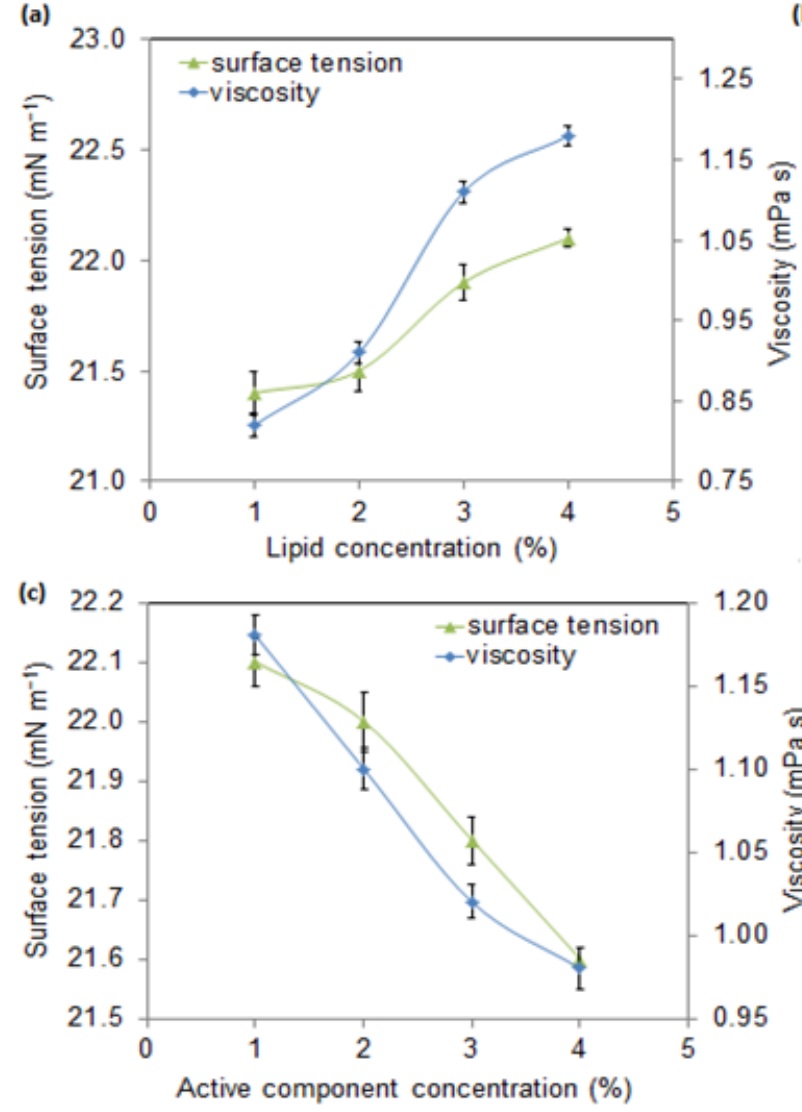

(b)
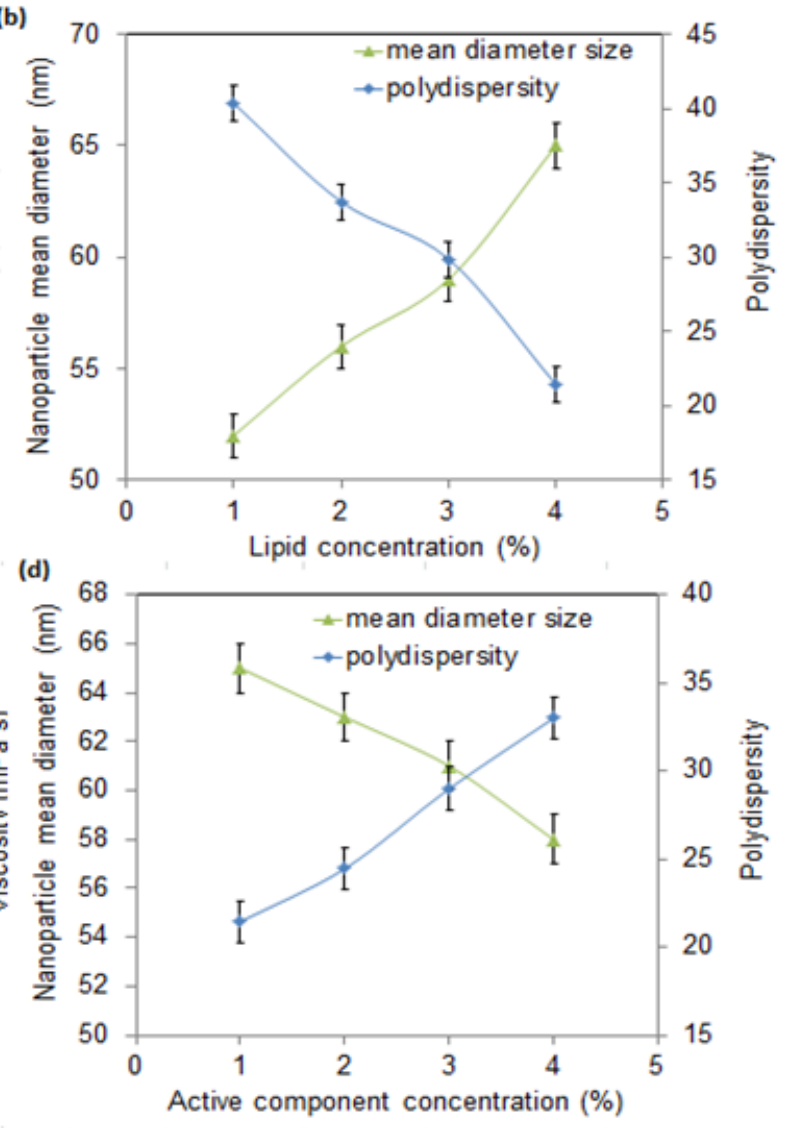

Figure 2: (a) Effect of lipid concentration, surface tension and viscosity of solution, (b) mean diameter and polydispersity of nanoparticles prepared at different stearic acid lipid concentrations. Effect of EV weight concentration (wt\%) on $4 \mathrm{wt} \%$ of lipid solution on: (c) surface tension, and viscosity, (d) mean diameter and polydispersity. $N \simeq 300$ nanoparticles.

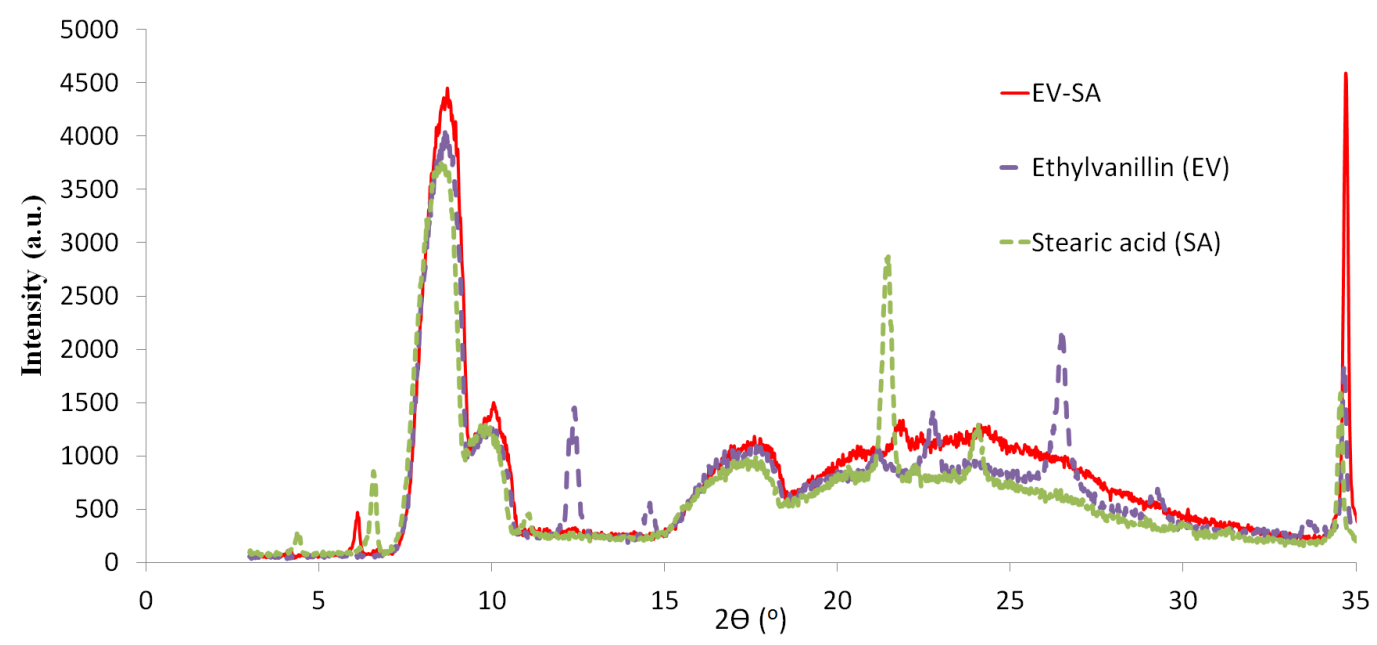

Figure 3: Comparison of the XRD curves of pure EV, pure SA and EV with SA nanoparticles, 4 wt\% SA nanoparticles containing $1.6 \mathrm{wt} \% \mathrm{EV}$, prepared at a flow rate $15 \mu \mathrm{l} \mathrm{min}^{-1}$ and applied voltage $14.5 \mathrm{kV}$. All curves were recorded with the same temperature calibration. On the Intensity axis, a.u. indicates arbitrary units. 


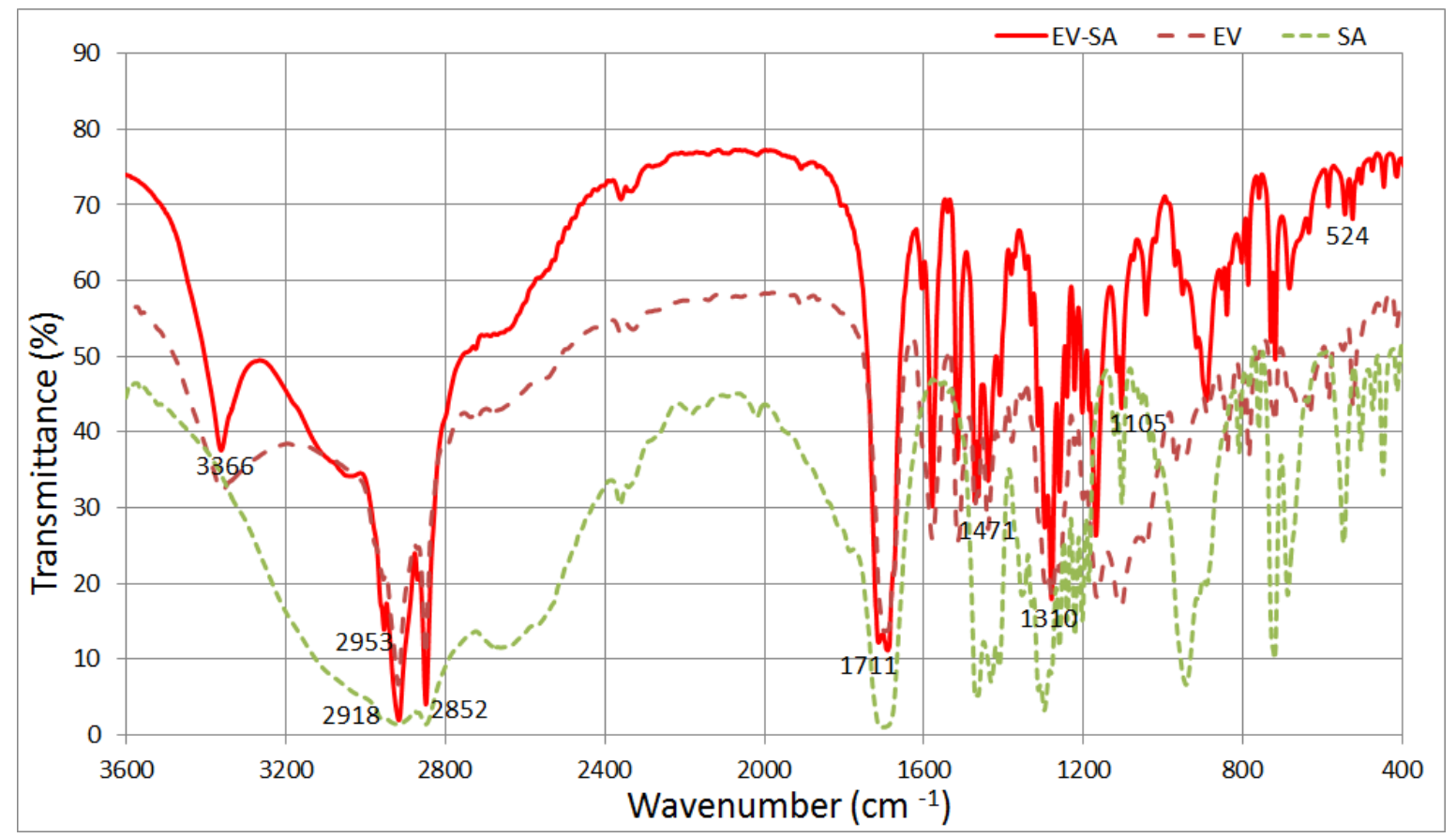

Figure 4: FT-IR spectra of pure EV, pure SA and EV with SA nanoparticles, 4 wt\% SA nanoparticles containing $1.6 \mathrm{wt} \%$ EV, prepared at flow rate $15 \mu \mathrm{lmin}^{-1}$ and applied voltage $14.5 \mathrm{kV}$. The nanoparticle spectra show peaks that are characteristic of both pure EV and pure SA.

Table 1: Model parameters for food component release from nanoparticles. The percentages of ethylvanillin (EV) and stearic acid (SA) are shown, together with the average diameters of the nanoparticles $\langle d\rangle$. Values of AICc, $R_{\mathrm{adj}}^{2}$ and $R^{2}$ are reported for the models represented by equation 8 (tanh) and equation 4 (First Order).

\begin{tabular}{cccc|ccc|ccc}
\hline & & & & \multicolumn{3}{|c|}{ tanh } & \multicolumn{3}{|c}{ First Order } \\
\hline Case & EV wt\% & SAwt $\%$ & $\langle d\rangle / \mathrm{nm}$ & AICc & $R_{\text {adj }}^{2}$ & $R^{2}$ & AICc & $R_{\text {adj }}^{2}$ & $R^{2}$ \\
\hline S1 & 1 & 1 & 52 & 75.3 & 0.998 & 0.998 & 93.0 & 0.992 & 0.993 \\
S2 & 1 & 2 & 56 & 80.8 & 0.996 & 0.997 & 97.5 & 0.987 & 0.989 \\
S3 & 1 & 3 & 59 & 78.6 & 0.997 & 0.997 & 94.3 & 0.989 & 0.990 \\
S4 & 1 & 4 & 65 & 88.5 & 0.992 & 0.993 & 87.2 & 0.992 & 0.994 \\
S6 & 2 & 4 & 63 & 89.3 & 0.992 & 0.993 & 87.8 & 0.993 & 0.994 \\
S7 & 3 & 4 & 61 & 87.3 & 0.993 & 0.994 & 88.0 & 0.993 & 0.994 \\
S8 & 4 & 4 & 58 & 85.1 & 0.996 & 0.996 & 73.8 & 0.998 & 0.998 \\
\hline
\end{tabular}



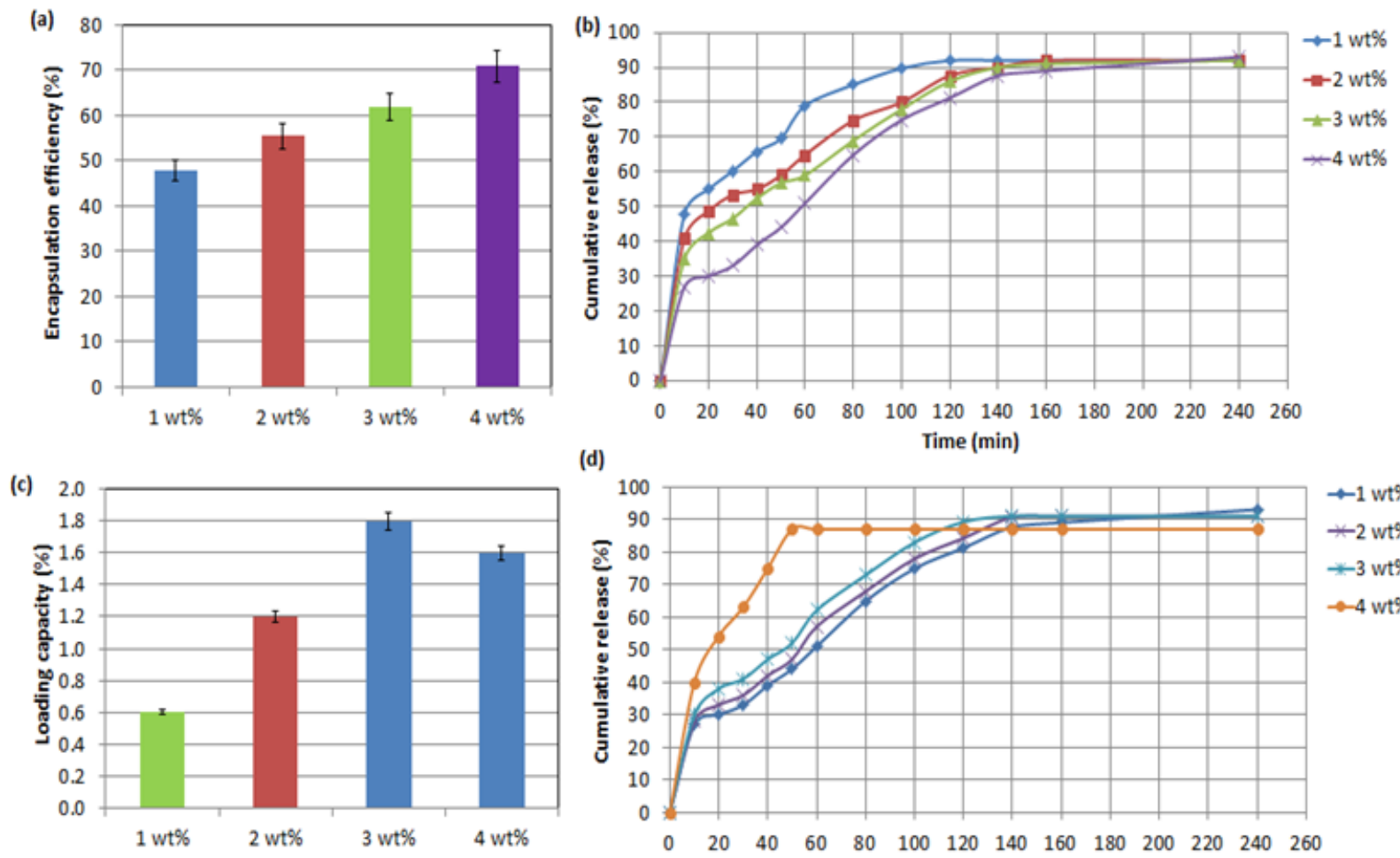

(d)

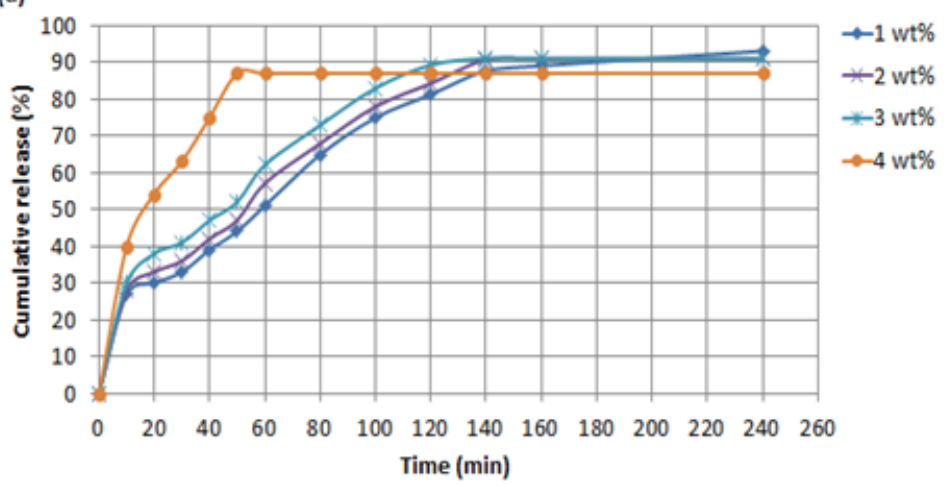

Figure 5: (a) EV encapsulation efficiency of lipid nanoparticles prepared from solutions 1, 2, 3 and 4 wt\% of the SA, (b) EV release profile for lipid nanoparticles prepared from solutions 1, 2, 3 and $4 \mathrm{wt} \%$ of the SA, (c) EV loading capacity of lipid nanoparticles at various initial flavour component inputs ranging from 1 to $4 \mathrm{wt} \%$ of the EV, and (d) EV release profile for nanoparticles prepared from 1 to $4 \mathrm{wt} \%$ of the EV solutions. 
This suggest diffusional release from a spherical capsule with a lipid layer thickness in close agreement with what we observed. A diffusion constant of this magnitude is broadly comparable with the value of $5-7 \times 10^{-20} \mathrm{~m}^{2} \mathrm{~s}^{-1}$ observed[68] for lidocaine in polylactic acid particles with sizes in the range 250 to $800 \mathrm{~nm}$. We conclude that diffusion is the determining factor in the release rate. We intend to undertake more detailed numerical modelling, including variation of the capsule shell properties with time, in order to address the two-stage behaviour apparent in the release rate curves.

\section{Conclusions}

We have demonstrated that lipid nanoparticles encapsulating a model active component (ethylvanillin) can be produced by electrospraying. We found that the diameter and size distribution of the nanoparticles could be controlled by varying the lipid:ethylvanillin concentration ratio in the sprayed solution. The nanoparticles were found to have a core-shell structure, with the thickness of the outer lipid layer being dependent upon the concentration of lipid in the processed solution. The encapsulation efficiency and loading capacity were similarly found to be dependent on the lipid: ethylvanillin concentration ratio; although there was found to be a limit above which both started to decrease. It was shown that the release of the ethylvanillin was consistent with diffusion through a lipid membrane. A new release model incorporating a tanh function provided the best overall fit to the time dependence of the release of ethylvanillin from the nanoparticles, but further analysis suggests that a first-order release model is more applicable. The electrohydrodynamic technique for preparation of lipid nanoparticles in one step may be very appropriate for mass production of lipid based nanoparticles with different characteristics for food or medical applications.

\section{References}

[1] P. Sanguansri, M. A. Augustin, Nanoscale materials development - a food industry perspective, Trends in Food Science and Technology 17 (10) (2006) 547-556. doi:10.1016/j.tifs.2006.04.010.

[2] M. Fathi, M. Mohebbi, Increasing food safety using nanotechnology, Magazine of Nanotechnology Initiative Council (2010) 16-18.
[3] S. Neethirajan, D. S. Jayas, Nanotechnology for the Food and Bioprocessing Industries, Food and Bioprocess Technology 4 (2011) 39-47. doi:10.1007/s11947010-0328-2.

[4] H. D. Chen, J. C. Weiss, F. Shahidi, Nanotechnology in nutraceuticals and functional foods, Food Technology 60 (2006) 30-36.

[5] E. Shimoni, Nanotechnology for Foods: Delivery Systems, in: G. Barbosa-Cánovas, A. Mortimer, D. Lineback, W. Spiess, K. Buckle, P. Colonna (Eds.), Global Issues in Food Science and Technology, Academic Press: San Diego, 2009, pp. 411-424.

[6] O. C. Farokhzad, R. Langer, Impact of nanotechnology on drug delivery, ACS Nano 3 (2009) 16-20. doi:10.1021/nn900002m.

[7] J. Liu, T. Gong, H. Fu, C. Wang, X. Wang, Q. Chen, Q. Zhang, Q. He, Z. Zhang, Solid lipid nanoparticles for pulmonary delivery of insulin, International Journal of Pharmaceutics 356 (1-2) (2008) 333-344. doi:10.1016/j.ijpharm.2008.01.008.

[8] R. H. Müller, R. D. Petersen, A. Hommoss, J. Pardeike, Nanostructured lipid carriers (NLC) in cosmetic dermal products, Advanced Drug Delivery Reviews 59 (6) (2007) 522-530. doi:10.1016/j.addr.2007.04.012.

[9] M. R. Mozafari, J. Flanagan, L. Matia-Merino, A. Awati, A. Omri, Z. E. Suntres, H. Singh, Recent trends in the lipid-based nanoencapsulation of antioxidants and their role in foods, Journal of the Science of Food and Agriculture 86 (13) (2006) 2038-2045. doi:10.1002/jsfa.2576.

[10] D. J. McClements, E. A. Decker, Y. Park, J. Weiss, Structural design principles for delivery of bioactive components in nutraceuticals and functional foods, Critical Reviews in Food Science and Nutrition 49 (6) (2009) 577-606. doi:10.1080/10408390902841529.

[11] K. P. Velikov, E. Pelan, Colloidal delivery systems for micronutrients and nutraceuticals, Soft Matter 4 (10) (2008) 1964-1980. doi:10.1039/B804863K.

[12] J. Weiss, E. A. Decker, D. J. McClements, K. Kristbergsson, T. Helgason, T. Awad, Solid lipid nanoparticles as delivery systems for bioactive food components, Food Biophysics 3 (2) (2008) 146-154. doi:10.1007/s11483-008-9065-8.

[13] P. S. Given, Jr, Encapsulation of Flavors in Emulsions for Beverages, Current Opinion in Colloid \& Interface Science 14 (1) (2009) 43-47. doi:10.1016/j.cocis.2008.01.007.

[14] L. Sagalowicz, M. E. Leser, Delivery systems for liquid food products, Current Opinion in Colloid \& Interface Science 15 (1-2) (2010) 61-72. doi:10.1016/j.cocis.2009.12.003.

[15] I. Lacatusu, E. Mitrea, N. Badea, R. Stan, O. Oprea, A. Meghea, Lipid nanoparticles based on omega3 fatty acids as effective carriers for lutein delivery. Preparation and in vitro characterization studies, Journal of Functional Foods 5 (3) (2013) 1260-1269. doi:10.1016/j.jff.2013.04.010.

[16] M. Fathi, J. Varshosaz, Novel hesperetin loaded nanocarriers for food fortification: Production and characterization, Journal of Functional Foods 5 (3) (2013) 1382-1391. doi:10.1016/j.jff.2013.05.006.

[17] I. Stratulat, M. Britten, S. Salmieri, P. Fustier, D. St-Gelais, C. P. Champagne, M. Lacroix, Enrichment of cheese with bioactive lipophilic compounds, Journal of Functional Foods 6 (1) (2014) 48-59. 
doi:10.1016/j.jff.2013.11.023.

[18] Y. Ting, Y. Jiang, C.-T. Ho, Q. Huang, Common delivery systems for enhancing in vivo bioavailability and biological efficacy of nutraceuticals, Journal of Functional Foods 7 (1) (2014) 112-128. doi:10.1016/j.jff.2013.12.010.

[19] W. Liu, A. Ye, W. Liu, C. Liu, H. Singh, Liposomes as food ingredients and nutraceutical delivery systems, Agro Food Industry Hi-Tech 24 (2013) 68-71.

[20] B. C. Keller, Liposomes in nutrition, Trends in Food Science \& Technology 12 (1) (2001) 25-31. doi:10.1016/S0924-2244(01)00044-9.

[21] P. da Silva Malheiros, D. J. Daroit, A. Brandelli, Food applications of liposome-encapsulated antimicrobial peptides, Trends in Food Science \& Technology 21 (6) (2010) 284-292. doi:10.1016/j.tifs.2010.03.003.

[22] S. Madrigal-Carballo, S. Lim, G. Rodriguez, O. Vila, C. G. Krueger, S. Gunasekaran, Biopolymer coating of soybean lecithin liposomes via layer-by-layer selfassembly as novel delivery system for ellagic acid, Journal of Functional Foods 2 (2) (2010) 99-106. doi:10.1016/j.jff.2010.01.002.

[23] S. P. Vyas, S. Rai, R. Paliwal, P. N. Gupta, K. Khatri, A. K. Goyal, Solid Lipid Nanoparticles (SLNs) as a Rising Tool in Drug Delivery Science: One Step Up in Nanotechnology, Current Nanoscience 4 (1) (2008) 30-44. doi:10.2174/157341308783591816.

[24] R. H. Müller, K. Mäder, S. Gohla, Solid lipid nanoparticles (SLN) for controlled drug delivery - A review of the state of the art, European Journal of Pharmaceutics and Biopharmaceutics 50 (1) (2000) 161-177. doi:10.1016/S0939-6411(00)00087-4.

[25] W. Mehnert, K. Mäder, Solid lipid nanoparticles - Production, characterization and applications, Advanced Drug Delivery Reviews 47 (2-3) (2001) 165-196. doi:10.1016/S0169-409X(01)00105-3.

[26] C. Schwarz, W. Mehnert, J. Lucks, R. Müller, Solid lipid nanoparticles (SLN) for controlled drug delivery. I. Production, characterization and sterilization, Journal of Controlled Release 30 (1) (1994) 83-96. doi:10.1016/0168-3659(94)90047-7.

[27] A. zur Mühlen, C. Schwarz, W. Mehnert, Solid lipid nanoparticles (SLN) for controlled drug delivery - Drug release and release mechanism, European Journal of Pharmaceutics and Biopharmaceutics 45 (2) (1998) 149-155. doi:10.1016/S0939-6411(97)00150-1.

[28] R. Cavalli, O. Caputo, M. R. Gasco, Solid lipospheres of doxorubicin and idarubicin, International Journal of Pharmaceutics 89 (1) (1993) R9-R12. doi:10.1016/0378-5173(93)90313-5.

[29] H. Heiati, R. Tawashi, N. C. Phillips, Drug retention and stability of solid lipid nanoparticles containing azidothymidine palmitate after autoclaving, storage and lyophilization, Journal of Microencapsulation 15 (2) (1998) 173-184. doi:10.3109/02652049809006847.

[30] R. H. Müller, M. Radtke, S. A. Wissing, Solid lipid nanoparticles (SLN) and nanostructured lipid carriers (NLC) in cosmetic and dermatological preparations, Advanced Drug Delivery Reviews 54 (Supplement) (2002) S131-S155. doi:10.1016/S0169-409X(02)001187.

[31] R. H. Müller, M. Radtke, S. A. Wissing, Nanostructured lipid matrices for improved microencapsulation of drugs, International Journal of Pharmaceutics 242 (1-2) (2002) 121-128. doi:10.1016/S0378-5173(02)00180-1.
[32] R. Cavalli, C. Bocca, A. Miglietta, O. Caputo, M. Gasco, 1999 Albumin adsorption on stealth and nonstealth solid lipid nanoparticles, STP pharma sciences 9 (1999) 183-189.

[33] E. B. Souto, S. A. Wissing, C. M. Barbosa, R. H. Müller, Development of a controlled release formulation based on SLN and NLC for topical clotrimazole delivery, International Journal of Pharmaceutics 278 (1) (2004) 71-77. doi:10.1016/j.ijpharm.2004.02.032.

[34] G. Kim, J. Park, H. Han, Production of microsized PMMA droplets using electrospraying with various auxiliary fields, Journal of Colloid and Interface Science 299 (2) (2006) 593-598. doi:10.1016/j.jcis.2006.02.054.

[35] D.-R. Chen, D. Y. Pui, S. L. Kaufman, Electrospraying of conducting liquids for monodisperse aerosol generation in the $4 \mathrm{~nm}$ to $1.8 \mu \mathrm{m}$ diameter range, Journal of Aerosol Science 26 (6) (1995) 963-977. doi:10.1016/0021-8502(95)00027-A.

[36] L. de Juan, J. F. de la Mora, Charge and size distributions of electrospray drops, Journal of Colloid and Interface Science 186 (2) (1997) 280-293. doi:10.1006/jcis.1996.4654.

[37] M. Enayati, Z. Ahmad, E. Stride, M. Edirisinghe, One-step electrohydrodynamic production of drug-loaded micro- and nanoparticles, Journal of the Royal Society Interface 7 (45) (2010) 667-675. doi:10.1098/rsif.2009.0348.

[38] M. Enayati, Z. Ahmad, E. Stride, M. Edirisinghe, Size mapping of electric field-assisted production of polycaprolactone particles, Journal of the Royal Society Interface 7 (Supplement 4) (2010) S393-S402. doi:10.1098/rsif.2010.0099.focus.

[39] D. Wallick, Cellulose Polymers in Microencapsulation of Food Additives, in: A. G. Gaonkar, N. Vasisht, A. R. Khare, R. Sobel (Eds.), Microencapsulation in the Food Industry, Academic Press: San Diego, 2014, Ch. 17, pp. 181-193.

[40] D.-G. Yu, X.-X. Shen, C. Branford-White, K. White, L.-M. Zhu, S. W. A. Bligh, Oral fast-dissolving drug delivery membranes prepared from electrospun polyvinylpyrrolidone ultrafine fibers, Nanotechnology 20 (5) (2009) 055104. doi:10.1088/09574484/20/5/055104

[41] D.-G. Yu, P. Lu, C. Branford-White, J.-H. Yang, X. Wang, Polyacrylonitrile nanofibers prepared using coaxial electrospinning with $\mathrm{LiCl}$ solution as sheath fluid, Nanotechnology 22 (43) (2011) 435301. doi:10.1088/0957-4484/22/43/435301.

[42] D. G. Yu, J. H. Yang, X. Wang, F. Tian, Liposomes self-assembled from electrosprayed composite microparticles, Nanotechnology 23 (10) (2012) 105606. doi:10.1088/0957-4484/23/10/105606.

[43] R. Pareta, M. J. Edirisinghe, A novel method for the preparation of biodegradable microspheres for protein drug delivery, Journal of the Royal Society Interface 3 (9) (2006) 573-582. doi:10.1098/rsif.2006.0120.

[44] U. Farook, E. Stride, M. J. Edirisinghe, Preparation of suspensions of phospholipid-coated microbubbles by coaxial electrohydrodynamic atomization, Journal of the Royal Society Interface 6 (32) (2009) 271-277. doi:10.1098/rsif.2008.0225.

[45] H. Valo, L. Peltonen, S. Vehviläinen, M. Karjalainen, R. Kostiainen, T. Laaksonen, J. Hirvonen, Electrospray Encapsulation of Hydrophilic and Hydrophobic Drugs in Poly(L-lactic acid) Nanoparticles, Small 5 (15) 
(2009) 1791-1798. doi:10.1002/smll.200801907.

[46] E. Stride, K. Pancholi, M. Edirisinghe, S. Samarasinghe, Increasing the nonlinear character of microbubble oscillations at low acoustic pressures, Journal of the Royal Society Interface 5 (24) (2008) 807-811. doi:10.1098/rsif.2008.0005.

[47] P. H. M. Böttger, Z. Bi, D. Adolph, K. A. Dick, L. S. Karlsson, M. N. A. Karlsson, B. A. Wacaser, K. Deppert, Electrospraying of colloidal nanoparticles for seeding of nanostructure growth, Nanotechnology 18 (10) (2007) 105304. doi:10.1088/0957-4484/18/10/105304.

[48] W. E. Teo, S. Ramakrishna, A review on electrospinning design and nanofibre assemblies, Nanotechnology 17 (14) (2006) R89-R106. doi:10.1088/09574484/17/14/R01.

[49] S. Prilutsky, E. Zussman, Y. Cohen, The effect of embedded carbon nanotubes on the morphological evolution during the carbonization of poly(acrylonitrile) nanofibers, Nanotechnology 19 (16) (2008) 165603. doi:10.1088/0957-4484/19/16/165603.

[50] M. Eltayeb, E. Stride, M. Edirisinghe, Electrosprayed core-shell polymer-lipid nanoparticles for active component delivery, Nanotechnology 24 (46) (2013) 465604. doi:10.1088/0957-4484/24/46/465604.

[51] M. Eltayeb, E. Stride, M. Edirisinghe, Preparation, characterization and release kinetics of ethylcellulose nanoparticles encapsulating ethylvanillin as a model functional component, Journal of Functional Foods 14 (2015) 726-735. doi:10.1016/j.jff.2015.02.036.

[52] M. D. Paine, M. S. Alexander, J. P. W. Stark, Nozzle and liquid effects on the spray modes in nanoelectrospray, Journal of Colloid and Interface Science. 305 (1) (2007) 111-123. doi:10.1016/j.jcis.2006.09.031.

[53] S. Labbaf, S. Deb, G. Cama, E. Stride, M. Edirisinghe, Preparation of multicompartment sub-micron particles using a triple-needle electrohydrodynamic device, Journal of Colloid and Interface Science 409 (2013) 245-254. doi:10.1016/j.jcis.2013.07.033.

[54] Z. Ahmad, H. B. Zhang, U. Farook, M. Edirisinghe, E. Stride, P. Colombo, Generation of multilayered structures for biomedical applications using a novel trineedle coaxial device and electrohydrodynamic flow, Journal of the Royal Society Interface 5 (27) (2008) 1255-1261. doi:10.1098/rsif.2008.0247.

[55] V. Manojlovic, N. Rajic, J. Djonlagic, B. Obradovic, V. Nedovic, B. Bugarski, Application of Electrostatic Extrusion - Flavour Encapsulation and Controlled Release, Sensors 8 (3) (2008) 1488-1496. doi: $10.3390 / \mathrm{s} 8031488$.

[56] M. Eltayeb, P. K. Bakhshi, E. Stride, M. Edirisinghe, Preparation of solid lipid nanoparticles containing active compound by electrohydrodynamic spraying, Food Research International 53 (1) (2013) 88-95. doi:10.1016/j.foodres.2013.03.047.

[57] T. Higuchi, Mechanism of sustained-action medication. Theoretical analysis of rate of release of solid drugs dispersed in solid matrices, Journal of Pharmaceutical Sciences 52 (12) (1963) 1145-1149. doi: $10.1002 / j p s .2600521210$.

[58] A. D. Sezer, H. Kazak, E. T. Öner, J. Akbuğa, Levan-based nanocarrier system for peptide and protein drug delivery: Optimization and influence of experimental parameters on the nanoparticle characteristics, Carbohydrate Polymers 84 (1) (2011) 358-363. doi:10.1016/j.carbpol.2010.11.046.
[59] P. L. Ritger, N. A. Peppas, A simple equation for description of solute release I. Fickian and non-Fickian release from non-swellable devices in the form of slabs, spheres, cylinders or discs, Journal of Controlled Release 5 (1) (1987) 23 - 36. doi:10.1016/01683659(87)90034-4.

[60] N. A. Peppas, Analysis of Fickian and non-Fickian drug release from polymers, Pharmaceutica Acta Helvetiae 60 (1985) 110-111.

[61] J. Doshi, D. H. Reneker, Electrospinning Process and Applications of Electrospun Fibers, Journal of Electrostatics 35 (2-3) (1995) 151-160. doi:10.1016/03043886(95)00041-8.

[62] Y. Xu, M. A. Hanna, Morphological and structural properties of two-phase coaxial jet electrosprayed BSAPLA capsules, Journal of Microencapsulation 25 (7) (2008) 469-477. doi:10.1080/02652040802049513.

[63] A. Jaworek, A. T. Sobczyk, Electrospraying route to nanotechnology: An overview, Journal of Electrostatics $66 \quad(3-4) \quad(2008)$ 197-219. doi:10.1016/j.elstat.2007.10.001.

[64] T. C. Rohner, N. Lion, H. H. Girault, Electrochemical and theoretical aspects of electrospray ionisation, Physical Chemistry Chemical Physics 6 (12) (2004) 30563068. doi:10.1039/B316836K.

[65] E. F. Vieira, A. R. Cestari, E. B. de Santos, C. X. Rezende, Measurement of cation binding to immobilized vanillin by isothermal calorimetry, Journal of Colloid and Interface Science 298 (1) (2006) 74-78. doi:10.1016/j.jcis.2005.12.021.

[66] A. C. González-Baró, B. S. Parajón-Costa, C. A. Franca, R. Pis-Diez, Theoretical and spectroscopic study of vanillic acid, Journal of Molecular Structure 889 (1-3) (2008) 204-210. doi:10.1016/j.molstruc.2008.01.049.

[67] D. Zheng, C. Hu, Y. Peng, S. Hu, A carbon nanotube/polyvanillin composite film as an electrocatalyst for the electrochemical oxidation of nitrite and its application as a nitrite sensor, Electrochimica Acta 54 (21) (2009) 4910-4915. doi:10.1016/j.electacta.2009.04.004.

[68] M. Polakovič, T. Görner, R. Gref, E. Dellacherie, Lidocaine loaded biodegradable nanospheres: II. Modelling of drug release, Journal of Controlled Release 60 (2-3) (1999) 169 - 177. doi:10.1016/S0168-3659(99)00012-7.

[69] R. W. Korsmeyer, R. Gurny, E. Doelker, P. Buri, N. A. Peppas, Mechanisms of solute release from porous hydrophilic polymers., International Journal of Pharmaceutics 15 (1) (1983) 25-35. doi:10.1016/03785173(83)90064-9.

[70] P. L. Ritger, N. A. Peppas, A simple equation for description of solute release II. Fickian and anomalous release from swellable devices, Journal of Controlled Release 5 (1) (1987) 37 - 42. doi:10.1016/01683659(87)90035-6.

[71] N. A. Peppas, J. J. Sahlin, A simple equation for the description of solute release. III. Coupling of diffusion and relaxation, International Journal of Pharmaceutics 57 (2) (1989) 169 - 172. doi:10.1016/03785173(89)90306-2.

[72] H. Carslaw, J. Jaeger, Conduction of heat in solids, 2nd Edition, Oxford, 1959 


\section{Appendix A. New Fitting Function}

The expression derived by Peppas and collaborators $[59,69-71]$ is frequently used to fit release rates, but it has the disadvantage that it is not applicable to the whole of the release. As a result some of the measurement data must be discarded in order to use the expression. Alternatively, the expression can be misused, and applied to the whole range of the release. Here we propose a one- or two-parameter expression which applies to the whole release, which is simple enough to be used for fitting to experimental data, and in which the parameters have clear physical interpretations.

We start from the same point as Peppas et al, and consider the release to take place by diffusion out of a spherical solid matrix with instant removal of the solute from the surface. That is, we solve the radially symmetrical equation for diffusion of the solute with concentration $C$

$$
\frac{\partial C(r, t)}{\partial t}=D\left(\frac{\partial^{2} C(r, t)}{\partial r^{2}}+\frac{2}{r} \frac{\partial C(r, t)}{\partial r}\right)
$$

with

$$
\begin{aligned}
& C(r, 0)=C_{0} \text { for } r \leq a \\
& C(a, t)=0 \text { for } r>a \text { for all } t,
\end{aligned}
$$

where $a$ is the sphere radius.

The solutions are well known (see, for example, the monograph by Carslaw and Jaeger[72]), and we may write for the average concentration inside the sphere at time $t$

$$
\begin{aligned}
C_{\mathrm{av}}(t)= & \frac{6 C_{0}}{\pi^{2}} \sum_{n=1}^{\infty} \frac{1}{n^{2}} \exp \left(-\frac{D n^{2} \pi^{2} t}{a^{2}}\right) \\
= & C_{0}\left[1-\frac{6(D t)^{1 / 2}}{a \pi^{2}}-\frac{3 D t}{a^{2}}\right. \\
& \left.+\frac{12(D t)^{1 / 2}}{a} \sum_{n=1}^{\infty} \operatorname{ierfc}\left(\frac{n a}{(D t)^{1 / 2}}\right)\right],
\end{aligned}
$$

where ierfc is the integral of the complementary error function. Equation A.4 is suitable for longer times, equation A.5 for short times. Of course, $C_{\mathrm{av}}(t) / C_{0}=1-Q(t) / Q_{0}$, where $Q(t)$ is the amount of the original loading $Q_{0}$ that has been released from the particle.

We make three observations: the fractional release depends only on one parameter, $(D t)^{1 / 2} / a$; the fractional release saturates for long times at a value of 1 ; the short-time release has the form $Q(t) / Q_{0} \approx 6(D t)^{1 / 2} /\left(\pi^{1 / 2} a\right)$. We find that

$$
Q_{\mathrm{a}}(t)=\tanh \left(\frac{6(D t)^{1 / 2}}{\pi^{1 / 2} a}\right)
$$

fits the exact expressions from Equations A.4 and A. 5 with a maximum error of 0.03 over the whole release range from $Q(t)=0$ to 1 . A marginally better fit, with a maximum error of 0.02 , can be obtained by using

$$
Q_{\mathrm{b}}(t)=\tanh \left(\frac{\beta(D t)^{1 / 2}}{a}\right)
$$

with $\beta=3.345$ (compare with $6 / \pi^{1 / 2}=3.385$ ). It is worth noting that fitting a power law of the form $B t^{n}$ to the first $60 \%$ release according to $Q_{\mathrm{b}}(t)$ gives $n=0.43$, which agrees with the power law fit to the exact expression over that range, as calculated by Peppas et al. Over the time range from zero to the time of $60 \%$ release as predicted by the exact solution of the diffusion equation the fitted tanh function deviates from the exact solution by a maximum of 0.03 , whereas at the end of that range $B t^{n}$ deviates by 0.04 . We note that for long times the exact solution varies exponentially as $\exp \left(-D \pi^{2} t / a^{2}\right)$ whereas the fitted function varies as $\exp \left(-2 \sqrt{\left(36 D t /\left(\pi a^{2}\right)\right)}\right)$ : although these forms are very different, the numerical differences in the range where these asymptotic forms apply would be below the resolution of practical experiments. The 'obvious' alternative saturating function which has an appropriate asymptotic form is $1-\exp (-\alpha t)$, but this has a maximum deviation from the exact release profile of 0.09 and overall is inferior to the expression given by either equation A.6 or A.7. 